\title{
Comparison of Motion-blurred Image Restoration Using Wiener Filter and Spatial Difference Technique
}

\author{
Baochen Jiang ${ }^{1,2}$, Aiping Yang ${ }^{1}$, Chengyou Wang ${ }^{2}$ and Zhengxin Hou ${ }^{1 *}$ \\ ${ }^{1}$ School of Electronic Information Engineering, Tianjin University, Tianjin 300072, \\ P. R. China \\ ${ }^{2}$ School of Mechanical, Electrical and Information Engineering, Shandong University, \\ Weihai 264209, P. R. China \\ jbc@sdu.edu.cn,yangaiping@tju.edu.cn,wangchengyou@sdu.edu.cn, \\ zhengxinhou@163.com
}

\begin{abstract}
Motion blur of image is caused by relative motion between camera and photographed object during protographing. Motion-blurred image restoration is an important part of the image restoration. Image restoration is the process of recovering original image from its degraded version. This paper focuses on image restoration blurred by uniform linear motion. Principle of image restoration technique is introduced. Degradation model and blurred model of horizontal motion are analyzed. Two methods to solve image restoration caused by uniform linear motion are described in detail: Wiener filter in frequency domain and difference method in spatial domain. Comparisons are made between spatial method and the method in frequency domain. Experimental results on test image show that the spatial difference technique has better performance than the method based on Wiener filter.
\end{abstract}

Keywords: image restoration; uniform linear motion; motion-blurred image; spatial difference technique; Wiener filter

\section{Introduction}

Image restoration is a main part of digital image processing, including research on restoration algorithms, and programming of some specific image processing problems. In the processes of image generation, transmission and recording, some distortion and variousdegree degradation occur inevitably. The purpose of image restoration is to remove degradation factors of degraded images, and recover it as far as possible. The quality of reconstructed images is judged not only by human subjective feeling, but also by some objective measures, such as squared error between reconstructed image and original image etc.

There are many degradation sources in the image system. Some degradation factors affect only some individual gray scale of the image, while some degradation factors blur a spatial area of the image. The former is called point degradation, and the latter is called spatial degradation. There are also degradations caused by digital, display, time, color, and chemical. This paper focuses on image restoration blurred by uniform linear motion.

Photos of running cars and flying airplanes are blurred, which is caused by motion of photographed objects. Similarly, if capturing a static object from the running car, the photo is also blurred, which is caused by motion of the camera. In sum, the image is blurred if there is enough relative motion between camera and photographed object during photographing. The 
phenomenon is called motion blur. It is a common problem of image processing, so research on motion-blurred image restoration is a main subject of image restoration.

Sondhi reviewed the techniques for digital restoration of images. Some examples of restoration were included to illustrate the methods discussed [1]. Fitton used Hough transform to extract linear features successfully from geoscientific images [2]. Many motion-blurred image restoration methods were proposed recent years, and the methods can be divided into two categories: methods in frequency domain and methods in spatial domain. Srivastava et al. proposed generalized partial differential equations based model to recover the original image from the blurred image in spatial domain [3]. Quan and Zhang built up the degradation model of motion blurred star image. Wiener filter with optimal window technique was adopted to deal with the motion-blurred images and its performance in restoration of image was discussed [4]. Li and Zhan compared several recovery algorithms and analyzed the reason for motion blur simulation images in [5]. The paper studies image restoration methods based on Wiener filter and spatial difference technique, simulates the two methods, and analyses simulation results.

The rest of this paper is organized as follows. Section 2 explains principle of image restoration technique, and introduces degradation model and blurred model of horizontal motion. Section 3 describes two restoration methods of motion-blurred images in detail: Wiener filter in frequency domain and difference method in spatial domain. Then Section 4 presents experimental results and comparisons with the above two methods. Finally, Conclusion and further work are given in Section 5.

\section{Principle of Image Restoration Technique}

\subsection{Degradation Model}

Motion blur of image is caused by relative motion between camera and photographed objects during photographing. In order to recover motion-blurred images, degradation model of images has to be built firstly, i.e., principle of image degradation has to be understood, analyzed, and expressed in mathematical model firstly. Because there are many reasons causing image degradation and the principle of image degradation is complex, it is complex and difficult to provide a perfect mathematical model. However, degraded systems can be described by linear model approximately in many common cases.

For example, $f(\xi, \eta)$ and $g(x, y)$ represent real image and degraded image respectively, and the relation between them is

$$
g(x, y)=\iint_{-\infty}^{\infty} f(\xi, \eta) h(x, y, \xi, \eta) \mathrm{d} \xi \mathrm{d} \eta+n(x, y),
$$

where $h(x, y, \xi, \eta)$ is point spread function (PSF), i.e., two-dimension impulse response function. $n(x, y)$ is noise interference called additive noise, as it is in the form of addition. The noise is called multiplicative noise if it is in form of multiplication.

Suppose that the point spread function is independent with the position of each point on the photographed object plane, then Eq. (1) can be expressed as

$$
g(x, y)=\iint_{-\infty}^{\infty} f(\xi, \eta) h(x-\xi, y-\eta) \mathrm{d} \xi \mathrm{d} \eta+n(x, y),
$$

where $h(x-\xi, y-\eta)$ is shift-invariant point spread function, and $h(x, y, \xi, \eta)$ in Eq. (1) is shift-variant point spread function. 
Image restoration is the process of getting the optimum estimation of $f(x, y)$ from the known degraded image $g(x, y)$, according to some prior information of $n(x, y), h(x, y, \xi, \eta)$ and $f(\xi, \eta)$.

\subsection{Blurred Model of Horizontal Motion}

Suppose the image $f(x, y)$ has a linear motion, $x_{0}(t)$ and $y_{0}(t)$ are the photographed object's component motions in $x$ axis direction and $y$ axis direction respectively, and the exposure time is $T$. Without considering other factors, the captured motion-blurred image $g(x, y)$ is

$$
g(x, y)=\int_{0}^{T} f\left[x-x_{0}(t), y-y_{0}(t)\right] \mathrm{d} t .
$$

Transform the above equation with Fourier transform, then

$$
G(u, v)=\int_{-\infty}^{\infty} \int_{-\infty}^{\infty} g(x, y) \mathrm{e}^{-\mathrm{j} 2 \pi(u x+v y)} \mathrm{d} x \mathrm{~d} y=\int_{0}^{T}\left[\int_{-\infty}^{\infty} \int_{-\infty}^{\infty} f\left[x-x_{0}(t), y-y_{0}(t)\right] \mathrm{e}^{-\mathrm{j} 2 \pi(u x+v y)} \mathrm{d} x \mathrm{~d} y\right] \mathrm{d} t .
$$

Exchange integral order and apply properties of Fourier transform, the above equation can be expressed as

$$
G(u, v)=F(u, v) \int_{0}^{T} \mathrm{e}^{-\mathrm{j} 2 \pi\left[u x_{0}(t)+v y_{0}(t)\right]} \mathrm{d} t .
$$

Define

$$
H(u, v)=\int_{0}^{T} \mathrm{e}^{-\mathrm{j} 2 \pi\left[u x_{0}(t)+v y_{0}(t)\right]} \mathrm{d} t,
$$

Then Eq. (5) can be expressed as

$$
G(u, v)=H(u, v) F(u, v),
$$

where $H(u, v)$ is called transfer function of motion blur. If properties of component motions $x(t)$ and $y(t)$ are known, the transfer function can be calculated with Eq. (6). When the image only has a uniform linear motion in $x$ axis direction with speed of $x_{0}(t)=\frac{c t}{T}$, then $y_{0}(t)=0$. Suppose $t=T$ and the total distance of image motion is $c$, Eq. (6) can be expressed as

$$
H(u, v)=\int_{0}^{T} \mathrm{e}^{-\mathrm{j} 2 \pi x_{0}(t)} \mathrm{d} t=\int_{0}^{T} \mathrm{e}^{-\mathrm{j} 2 \pi u \frac{c t}{T}} \mathrm{~d} t=\frac{T}{\pi u c} \sin (\pi u c) \mathrm{e}^{-\mathrm{j} 2 \pi u c} .
$$

If $n$ is an integer, the value of transfer function $H(u)$ at the point $u=\frac{n}{c}$ is 0 . The zero points of transfer function will cause big trouble when using inverse filtering restoration methods.

If the image only has a linear motion on $x$ axis direction, Eq. (3) can be expressed as 


$$
g(x)=\int_{0}^{T}\left[f\left(x-x_{0}(t)\right] \mathrm{d} t=\int_{0}^{T} f\left(x-\frac{c t}{T}\right) \mathrm{d} t .\right.
$$

\section{Motion-blurred Image Restoration}

Traditional motion-blurred image restoration methods are algebraic methods and frequency domain methods. Algebraic methods estimate the original image with preselected statistical criteria, and are used mainly in spatial domain. The frequency domain methods eliminate blur with various filters. Both algebraic methods and frequency domain methods have some disadvantages: algebraic methods cannot ensure the result image is the optimum estimation, while the frequency domain methods need to process the noise amplification caused by zero points of transfer function $H(u, v)$ during filtering [6]. Two restoration methods of motion-blurred images are introduced below: Wiener filter in frequency domain and difference method in spatial domain.

\subsection{Image Restoration based on Wiener Filter}

Wiener filter seeks transfer function $Y(\omega)$ which makes the minimum average variance between reconstructed target image estimation $\hat{F}(\omega)$ and real image, i.e.,

$$
\left\langle\int_{-\Omega}^{\Omega}|F(\omega)-\hat{F}(\omega)|^{2}\right\rangle=\min ,
$$

where

$$
\hat{F}(\omega)= \begin{cases}Y(\omega) \cdot G(\omega), & |\omega| \leq \Omega \\ 0, & |\omega|>\Omega\end{cases}
$$

Then solve the unconstrained extremum of Eq. (10). The transfer function of Wiener filter is [7]

$$
Y(\omega)=\frac{H^{*}(\omega)}{|H(\omega)|^{2}+P_{n}(\omega) / P_{f}(\omega)},
$$

where $H^{*}(\omega)$ is complex conjugate of degradation system's transfer function $H(\omega)$. $P_{f}(\omega)$ and $P_{n}(\omega)$ are target image and power spectrum density of the noise, respectively.

For two-dimension images, the transfer function of two-dimension Wiener filter is

$$
Y(u, v)=\frac{H^{*}(u, v)}{|H(u, v)|^{2}+P_{n}(u, v) / P_{f}(u, v)},
$$

then

$$
\hat{F}(u, v)=Y(u, v) \cdot G(u, v)=\frac{H^{*}(u, v) \cdot G(u, v)}{|H(u, v)|^{2}+P_{n}(u, v) / P_{f}(u, v)} .
$$

According to above description, steps of Wiener filtering algorithm are: 
Step 1. Solve the two-dimension Fourier transform $G(u, v)$ of degraded image $g(x, y)$, as follows

$$
G(u, v)=\sum_{x=0}^{N-1} \sum_{y=0}^{N-1} g(x, y) \mathrm{e}^{\frac{-\mathrm{j} 2 \pi}{N}(u x+v y)} .
$$

Step 2. Solve the two-dimension Fourier transform $H(u, v)$ of point spread function $h(u, v)$, as follows

$$
G(u, v)=\sum_{x=0}^{N-1} \sum_{y=0}^{N-1} h(x, y) \mathrm{e}^{\frac{-\mathrm{j} 2 \pi}{N}(u x+v y)} .
$$

Step 3. Calculate the target image $P_{f}(u, v)$ and power spectrum density of the noise $P_{n}(u, v)$.

Step 4. Calculate the target image's spectrum estimation $\hat{F}(u, v)$, as shown in Eq. (14).

Step 5. Solve the two-dimension inverse Fourier transform of $\hat{F}(u, v)$, i.e., obtain the reconstructed target image $\hat{f}(x, y)$, as follows

$$
\hat{F}(u, v)=G(u, v) / H(u, v) .
$$

Wiener filter has following characteristics:

(a) Different from inverse filter, Wiener filter doesn't exist ill-conditioned problem. It can be inferred form Eq. (14): when value of $H(u, v)$ is very small or zero, the denominator of Eq. (14) will not be zero and approximate $P_{n}(u, v) / P_{f}(u, v)$.

(b) If the noise approximates zero, i.e., $P_{n}(u, v) \rightarrow 0$, Eq. (14) can be expressed as

$$
\hat{F}(u, v) \rightarrow \frac{G(u, v)}{H(u, v)} .
$$

In other words, when the noise is very small or zero, Wiener filter approximates inverse filter.

(c) If the power spectrum approximates zero, i.e. $P_{f}(u, v) \rightarrow 0$, Eq. (14) can be expressed as

$$
\hat{F}(u, v) \rightarrow 0
$$

It is obviously a reasonable, because any meaningful target signals cannot be recovered from complete noise signals.

(d) If the transfer function $H(u, v)=1$, Eq. (14) can be expressed as

$$
F(u, v)=\frac{G(u, v)}{1+P_{n}(u, v) / P_{f}(u, v)} .
$$

Image restoration method based on Wiener filter is better restoration method. It can get satisfactory results in most cases. However, when the SNR is very small, the reconstructed result is unsatisfactory. This is caused by following reasons: 
(a) Wiener filter suppose that the system is a linear system. In fact, image records and human visual system evaluating images are nonlinear.

(b) Wiener filter is designed as an optimum filter according to minimum MSE criterion which may be not consistent with human vision evaluation criterion.

(c) Wiener filter is a stationary stochastic process model which may be not consistent with real images. In addition, Wiener filter just considers the covariance information of images, and ignores much other useful information.

In sum, Wiener filter can get better reconstructed results on two conditions that real images satisfy stationary stochastic process model and the degradation system is linear.

\subsection{Image Restoration using Spatial Difference Technique}

Suppose the original image $f(x, y)$ is zero or known outside the region $0 \leq x \leq L$. The original image $f(x, y)$ can be recovered by the difference of the region-inside blurred image $g(x, y)$, and it is called difference image restoration method [8].

Suppose the image only has a uniform linear motion in $x$ axis direction with speed of $x_{0}(t)=c t / T$, where $c$ is a constant, then Eq. (9) can be expressed as

$$
g(x)=\int_{0}^{T}\left[f\left(x-x_{0}(t)\right] \mathrm{d} t=\int_{0}^{T} f(x-c t / T) \mathrm{d} t=\int_{x-c}^{x} f(\tau) \mathrm{d} \tau, \quad 0 \leq x \leq L,\right.
$$

where $\tau=x-c t / T$. Solver the derivative of above equation and get an iterative formula as follows

$$
g^{\prime}(x)=f(x)-f(x-c), 0 \leq x \leq L,
$$

or

$$
f(x)=g^{\prime}(x)+f(x-c), 0 \leq x \leq L .
$$

For convenience, define $L=K c$, where $K$ is an integer, and define $p(z)$ is the part of image inside $0 \leq z \leq c$ during capturing:

$$
p(z)=f(z-c), 0 \leq z \leq c .
$$

It can be proved that Eq. (23) can be expressed as:

$$
f(x)=\sum_{k=0}^{m} g^{\prime}(x-k c)+p(x-m c), 0 \leq x \leq L,
$$

where $m$ is the integer part of $x / c$, and $g^{\prime}(x)$ is known. In order to get $f(x)$, only $p(x)$ needs to be estimated. A method estimating $p(x)$ directly from blurred image is introduced following.

Define

$$
\tilde{f}(x)=\sum_{j=0}^{m} g^{\prime}(x-j c),
$$

substitute it in Eq. (25): 


$$
p(x-m c)=f(x)-\tilde{f}(x) .
$$

If the value of $x$ is between 0 and $L$, then the value of $m$ varies from 0 to $K-1$. Then $p$ repeats $K$ times when independent variable's value of $f(x)$ varies from 0 to $L$. Solve Eq. (26) for each $k c \leq x<(k+1) c$, and sum up results when $k=0,1,2, \cdots, K-1$. When $0 \leq x<c$, $m=0$, then

$$
p(x)=\frac{1}{K} \sum_{k=0}^{K-1} f(x+k c)-\frac{1}{K} \sum_{k=0}^{K-1} \tilde{f}(x+k c) .
$$

The first item on right side of Eq. (28) is unknown, and approximates the mean value of $f(\cdot)$ when $K$ is very big. Define it as a constant $A$, and substitute Eq. (26) in Eq. (28), then

$$
p(x-m c) \approx A-\frac{1}{K} \sum_{k=0}^{K-1} \tilde{f}(x+k c-m c) \approx A-\frac{1}{K} \sum_{k=0}^{K-1} \sum_{j=0}^{k} g^{\prime}(x+k c-m c-j c),
$$

where $0 \leq x \leq L$. With Eq. (26) and Eq. (27), the following equation can be got.

$$
f(x) \approx A-\frac{1}{K} \sum_{k=0}^{K-1} \sum_{j=0}^{k} g^{\prime}(x+k c-m c-j c)+\sum_{j=0}^{m} g^{\prime}(x-j c) .
$$

Substitute $y$ in Eq. (30), and the final result is obtained.

$$
f(x, y) \approx A-\frac{1}{K} \sum_{k=0}^{K-1} \sum_{j=0}^{k} g^{\prime}(x+k c-m c-j c, y)+\sum_{j=0}^{m} g^{\prime}(x-j c, y) .
$$

The above algorithm is difference motion-blurred image restoration method [8].

\section{Experimental Results}

In this section, experiments in frequency domain methods and spatial domain methods are simulated. Two algorithms, Wiener filter restoration method in frequency domain and difference restoration method in spatial domain, are selected to simulate restoration of typical test images in MATLAB 7.0.

\subsection{Simulation of Wiener Filter Restoration Method}

Figure 1(a) is the fray image Cameraman whose gray scale is 256 and size is $256 \times 256$. Figure 1(b) and (c) are the blurred image which is shifted by 8 pixels in the horizontal direction and its reconstructed image using Wiener filter method, respectively. Figure 1(d) and (e) are the blurred image which is shifted by 32 pixels in the horizontal direction and its reconstructed image using Wiener filter method, respectively. Figure 1(f) and (g) are the blurred image which is shifted by 64 pixels in the horizontal direction and its reconstructed image using Wiener filter method, respectively. 


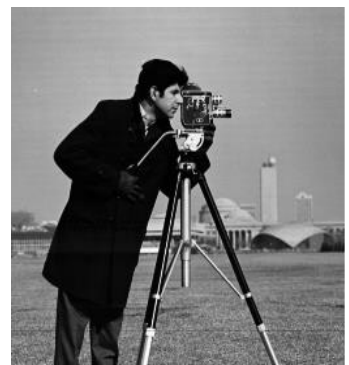

(a)

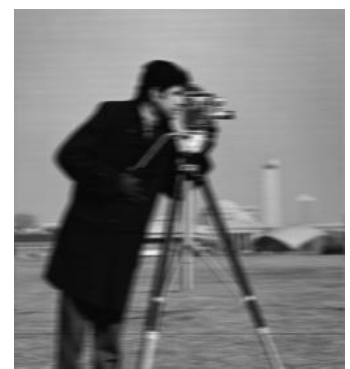

(b)

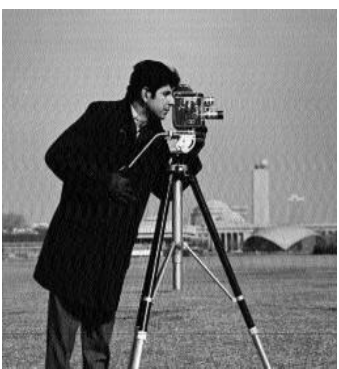

(c)

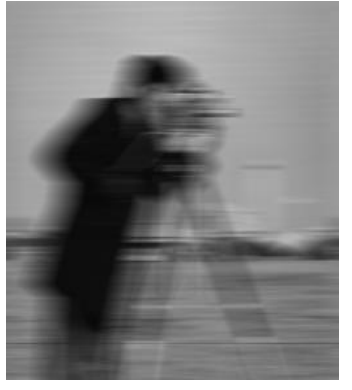

(d)

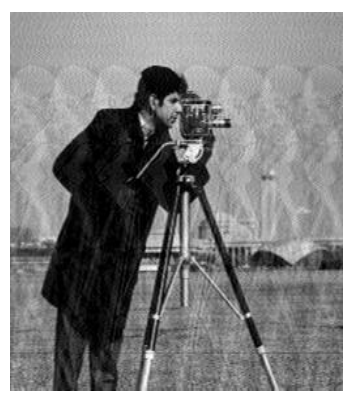

(e)

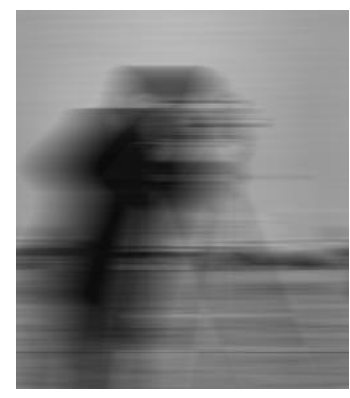

(f)

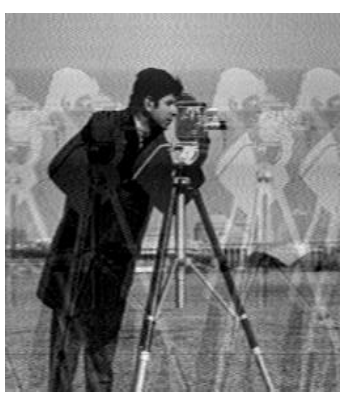

(g)

Figure 1. Restoration by Wiener Filter: (a) Original Image, (b) Blurred Image by Shifting 8 Pixels, (c) Reconstructed Image, (d) Blurred Image by Shifting 32 Pixels, (e) Reconstructed Image, (f) Blurred Image by Shifting 64 Pixels, (g) Reconstructed Image

It can be concluded from Figure 1 that during exposure, the bigger the relative shift between object and camera is, the more the lost data of blurred image is, so the reconstructed effect is worse. In other words, the restoration precision is inversely proportional to the relative shift between object and camera.

\subsection{Simulation of Difference Restoration Method}

The simulation of difference method uses the same test gray image Cameraman with size of $256 \times 256$. Figure 2 (a) and (b) are the blurred image which is shifted by 8 pixels in the horizontal direction and its reconstructed image using difference method, respectively. Figure 2(c) and (d) are the blurred image which is shifted by 80 pixels in the horizontal direction and its reconstructed image using difference method, respectively.

It can be concluded from Figure 2 that for difference restoration method, the restoration precision is also inversely proportional to the relative shift between object and camera. Neither frequency domain methods nor spatial domain methods can recover lost information of blurred images completely. If the relative shift between object and camera is very large, the reconstructed effect whether using any restoration method is very poor. 


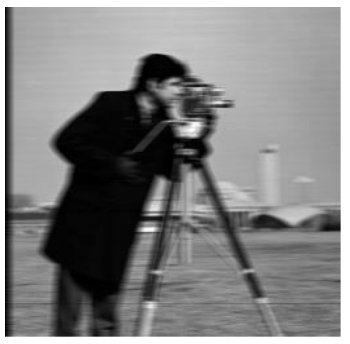

(a)

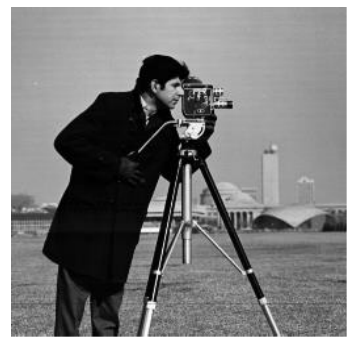

(b)

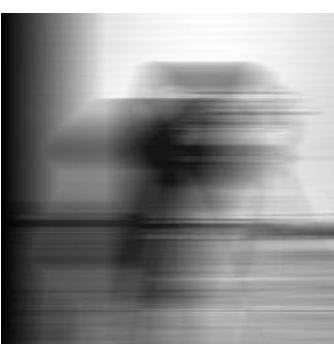

(c)

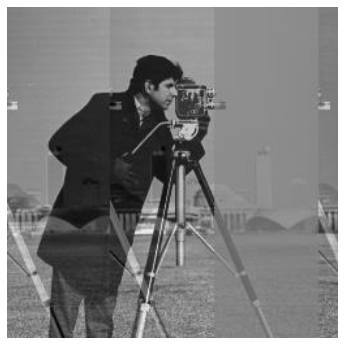

(d)

Figure 2. Restoration by Spatial Difference Technique: (a) Blurred Image by Shifting 8 Pixels, (b) Reconstructed Image, (c) Blurred Image by Shifting 80 Pixels, (d) Reconstructed Image

\subsection{Discussion}

With comparisons of the two restoration methods, it can be concluded that the reconstructed effect of spatial domain methods is better than frequency domain methods. There are two reasons causing worse effect of frequency domain methods:

(a) Strong pertinence. The frequency domain methods have their own application scope, because they are sensitive to frequency components of images.

(b) Ringing effect. It is an inherent problem of frequency domain process. The reason is frequency discontinuity caused by no solution of the equation near cut-off frequency.

In real applications, fuzzy parameters should be estimated as optimum as possible when they are uncertain. Without accurate or approximate parameters, the effect of reconstructed image is as poor as Figure 3.

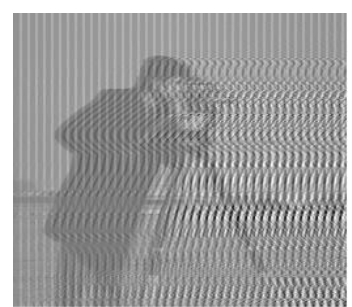

\section{Figure 3. Restoration Effect when Fuzzy Parameter is Not Accurate}

The paper supposes accurate fuzzy parameters are known, the work is just image restoration from known fuzzy parameters. However, in real applications, fuzzy parameters' estimation needs some prior knowledge, and it is difficult to get accurate fuzzy parameters. The paper doesn't introduce estimation of fuzzy parameters, which is the future work.

\section{Conclusion}

Motion-blurred image restoration methods are studied in this paper. The paper simulates restoration methods based on Wiener filter and spatial difference technique, and analyses simulation results. The conclusion is that the effect of restoration methods in spatial domain is better than restoration methods in frequency domain.

The paper focuses on motions on horizontal direction, without considering motions on any other direction. The properties of uniform linear motion on any direction are the same, and 
the only difference is the inclination angle. There are two methods to solve motions on any direction: one is transforming it to horizontal direction by a transform such as rotation, the other is substituting partial derivative of $x$ with the directional derivative on the motion direction. This issue is our future work.

\section{Acknowledgements}

This work was supported by the National Natural Science Foundation of China (Grant No. 61201371, No. 61002027). The authors would like to thank Chunhui Guo and Xiaoyan Wang for their help and valuable suggestions to improve the presentation of the paper.

\section{References}

[1] M. M. Sondhi, "Image restoration: The removal of spatially invariant degradations", Proceedings of the IEEE, vol. 60, no. 7, (1972), pp. 842-853.

[2] N. C. Fitton and S. J. D. Cox, "Optimising the application of the Hough transform for automatic feature extraction from geoscientic images”, Computers \& Geosciences, vol. 24, no. 10, (1998), pp. 933-951.

[3] R. Srivastava, H. Parthasarthy, J. R. P. Gupta, and D. R. Choudhary, "Image restoration from motion blurred image using PDEs formalism", Proceedings of the IEEE International Advance Computing Conference, Patiala, India, (2009), March 6-7, pp. 61-64.

[4] W. Quan and W. N. Zhang, "Restoration of motion-blurred star image based on Wiener filter", Proceedings of the 4th International Conference on Intelligent Computation Technology and Automation, Shenzhen, China, (2011), March 28-29, pp. 691-694.

[5] B. Li and Z. H. Zhan, "Research on motion blurred image restoration", Proceedings of the 5th International Congress on Image and Signal Processing, Chongqing, China, (2012), October 16-18, pp. 1307-1311.

[6] J. W. Zheng and L. D. Cai, "Restoration of uniform linear motion blurred images", Journal of Jinan University (Natural Science), vol. 23, no. 3, (2002), pp. 42-45.

[7] X. H. Wang and R. C. Zhao, "Restoration of motion-blurred images", Proceedings of the 2nd International Conference on Image and Graphics, Hefei, China, vol. 4875, no. 1, (2002), August 16-18, pp. 413-421.

[8] Y. J. Zhang, "Image Engineering (I) - Image Processing (2nd Edition)", Beijing: Tsinghua University Press, (2006), pp. 200-208.

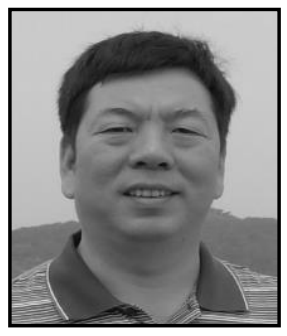

Baochen Jiang, was born in Shandong province, China in 1962. He received his B.S. degree in radio electronics from Shandong University, China, in 1983 and his M.E. degree in communication and electronic systems from Tsinghua University, China, in 1990. Now he is a professor in the School of Mechanical, Electrical and Information Engineering, Shandong University, Weihai, China. His current research interests include signal and information processing, image and video compression, and smart grid technology.

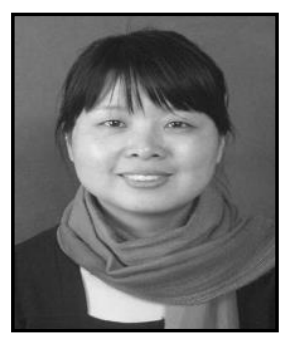

Aiping Yang, was born in Shandong province, China in 1976. She received her B.Ed. degree in mathematics education from Shandong Normal University, China, in 2000 and her M.S. degree in operations research and control theory from Tianjin University, China, in 2003 and her Ph.D. degree in signal and information processing from Tianjin University, China, in 2008. Now she is an associate professor in the School of Electronic Information Engineering, Tianjin University, China. Her current research interests include digital image and video processing, compressive sensing theory, and wavelet analysis. 


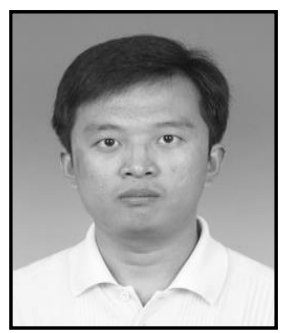

Chengyou Wang, was born in Shandong province, China in 1979. He received his B.E. degree in electronic information science and technology from Yantai University, China, in 2004 and his M.E. and Ph.D. degree in signal and information processing from Tianjin University, China, in 2007 and 2010 respectively. Now he is a lecturer in the School of Mechanical, Electrical and Information Engineering, Shandong University, Weihai, China. His current research interests include digital image and video processing, wavelet analysis and its applications, and smart grid technology.

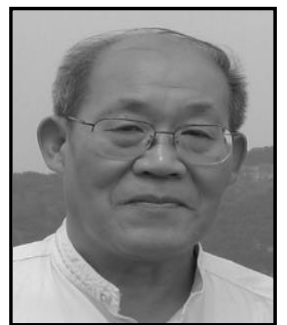

Zhengxin Houm, was born in Tianjin, China in 1945. He received his B.S. degree in radio electronics from Peking University, China, in 1969 and his M.E. degree in communication and electronic systems from Tianjin University, China, in 1982. Now he is a professor and supervisor of the Ph.D. students in the School of Electronic Information Engineering, Tianjin University, China. His current research interests include digital filtering theory, digital image and video processing, and wavelet analysis. 
International Journal of Signal Processing, Image Processing and Pattern Recognition Vol.7, No.2 (2014) 